\title{
Article
}

\section{Design of a surgical pen-type probe for real-time diagnosis of vascular flow using indocyanine green (ICG) fluorescence dye}

\author{
Ki-cheol Yoon 1,2, Kwang Gi Kim 1,2,3,4,*, and Seung Hoon Lee 5,6,
}

\begin{abstract}
${ }^{1}$ Dept. of Biomedical Engineering, College of Medicine, Gachon University, 38-13, Dokjom-ro 3, Namdong-gu, Incheon 21565, Republic of Korea; kcyoon98@gachon.ac.kr ${ }^{2}$ Medical Devices R\&D Center, Gachon University Gil Hospital, 21, 774 beon-gil, Namdong-daero Namdong-gu, Incheon 21565, Republic of Korea

${ }^{3}$ Dept. of Biomedical Engineering, College of Health Science, Gachon University, 191 Hambakmoero, Yeonsu-gu, Incheon 21936, Republic of Korea;

${ }^{4}$ Dept. of Health Sciences and Technology, Gachon Advanced Institute for Health Sciences and Technology (GAIHST), Gachon University, 38-13, 3 Dokjom-ro, Namdong-gu, Incheon 21565, Republic of Korea ${ }^{5}$ Dept. of Neurosurgery, Daejeon Eulji Medical Center (Eulji Univ. Hospital), Dunsanseo-ro, Seo-gu, Daejeon, 35233 Republic of Korea

${ }^{6}$ School of Medicine, Eulji University, 77 Gyeryong-ro 771 Beon-gil, Jung-gu Daejeon 34824 Republic of Korea

* Correspondence: kimkg@gachon.ac.kr; Tel.: +82-32-458-2770
\end{abstract}

\begin{abstract}
A surgical microscope is large in size, making portability impossible. The distance between the surgical microscope and the observation tissue is 15 to $30 \mathrm{~cm}$, while the maximum adjustment range of the camera to the right and left is $30^{\circ}$. Therefore, surgical microscopes cause attenuation (above 58\%) of the irradiation optical source owing to the long working distance. Moreover, the observation of tissue was dazzled with ambient light because the optical power source was strong ( 50 to $160 \mathrm{~mW} / \mathrm{cm}^{2}$ ). Owing to the limited ability to adjust the camera to the right and left, a blind spot occurs with a surgical microscope. Therefore, it is difficult to clearly observe a tumor.
\end{abstract}

In this study, a compact pen-type probe with a portable surgical microscope is proposed. The pen-type probe is small with a portable shape, and is capable of adjusting the working distance between itself and the observed tissue. It is also possible to adjust the viewing angle and fluorescence brightness. The proposed pen-type probe has no blind spots or optical density loss.

Keywords: fluorescence microscopy; fluorescence emission, malignant tumor, diagnosis, animal experiment

\section{Introduction}

Surgical diagnosis with indocyanine green (ICG) fluorescence dye is used to observe the flow conditions in blood vessels in the stomach, intestine, organ, liver, lymph node, and brain [1]-[5]. In particular, malignant tumors are complex and consist of tumors and blood vessels. Unfortunately, tumors and vasculature have the same color, making it difficult to identify the boundary between them with the naked eye. To observe the boundary between tumors and blood vessels, cancer surgery involves the of use surgical observation systems [2], such as surgical computed tomography (CT), surgical navigators, magnetic resonance imaging (MRI), positron emission tomography, ultrasonography, and surgical microscopes [6]-[12].

However, such systems mainly provide black and white images. Reading the size, location, and morphological boundaries of cerebrovascular, lymph node, or malignancies using a surgical microscope is difficult as the observations are not clear. The main reason for the poor image quality is because it is in black and white [8]. To solve this problem, a surgical color camera can be used, which show the color of the vascular and lymph 
nodes. However, because tumors and blood vessels have the same shape and color, it is difficult to distinguish and observe them using color cameras [13].

A fluorescence observation module can be connected to a surgical color-imaging camera. Fluorescent image guidance cameras for surgery can distinguish the shape, color, and boundaries of tumors and blood vessels because of their fluorescence expression [8], [14]. The disadvantages of such cameras are that they are large and heavy (camera head: above $4 \mathrm{~kg}$, whole: above $6 \mathrm{~kg}$ ) with limited portability. In addition, they allow limited adjustments of the observed beam angles (maximum $30^{\circ}$ ) and working distances (WD, 15-30 cm/ $\mathrm{cm}^{2}$ ) [15]-[20]. Because a surgical camera uses a high-energy light source $\left(50-160 \mathrm{~mW} / \mathrm{cm}^{2}\right)$ as the electromotive force of fluorescence emission, optical source beam energy loss (above 58\%) of the WD $(15-30 \mathrm{~cm}$ ) and strong ambient light around the tissue (photobleaching) can occur [10, 15]. In other words, the surgical microscope is difficult to apply in tissue observation because of the limited beam WD control and adjustment of the left and right of the camera [16, 17]. Moreover, surgical diagnostic systems are not portable because of their large size [10].

To solve the problems of the surgical fluorescence microscope, this study proposes a pen-type probe that is small and portable for real-time diagnosis. The performance of the proposed pen-type probe was evaluated through animal experiments using a light source of $785 \mathrm{~nm}$ and a fluorescence wavelength of $810 \mathrm{~nm}$.

\section{Fabrication of the Pen-type Probe}

The conventional surgical microscope (with a laser diode (LD) and near-infrared (NIR) camera) and the proposed pen-type probe were compared, as shown in Fig. 1 [18]. Fig. 2 shows the detailed structure of the proposed pen-type probe. As shown in the figure, the proposed pen-type probe consists of a LD, drive module (for laser drive), small camera module (SE-8J200), switch (ON/OFF), laser brightness control performance, battery, longpass filter, and communication cable. The communication cable connects the camera and the external monitor. The videos captured by the camera can be seen in real time on an external monitor through a cable.

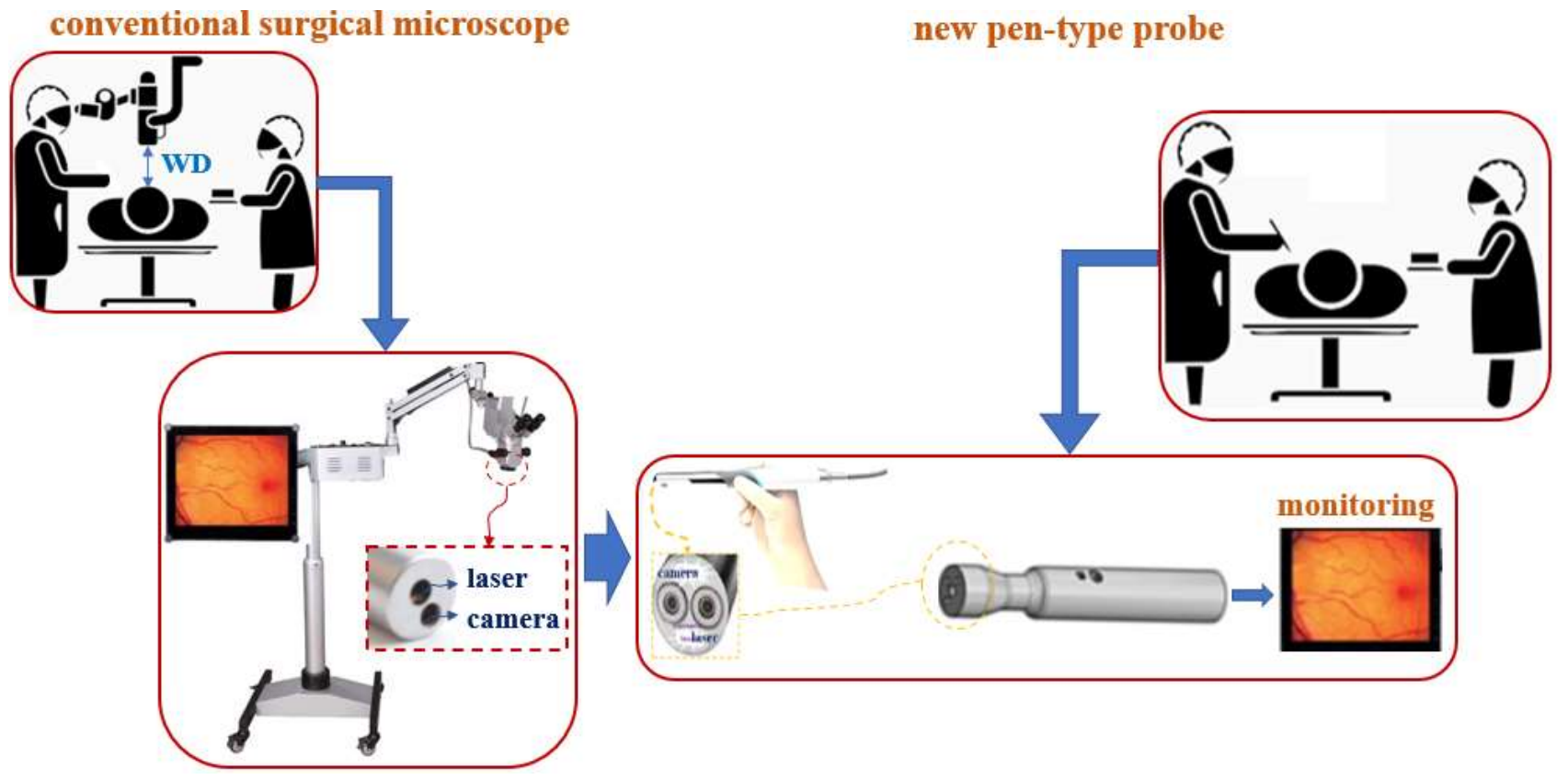

Figure 1. Structure of the conventional surgical microscope and the proposed pen-type probe. 


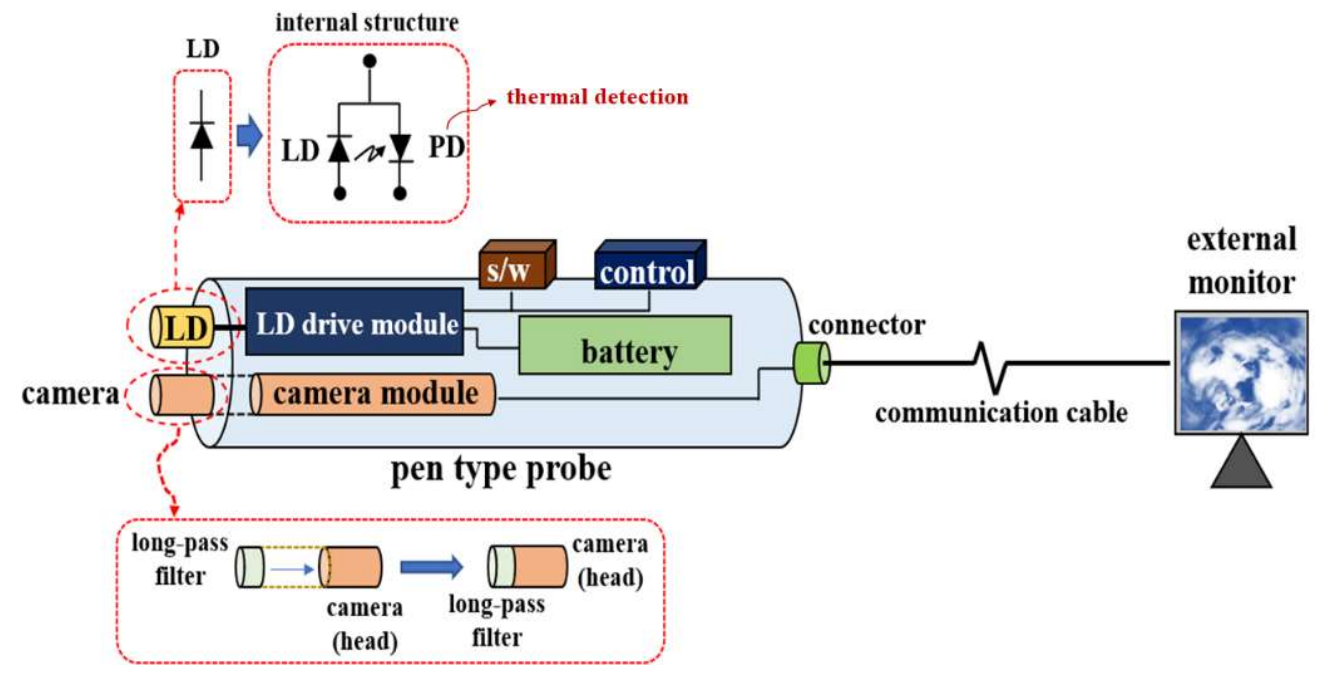

Figure 2. Detailed structure of the proposed pen-type probe.

The Thorlabs L785P090 LD was used, with excitation wavelength $\left(\lambda_{\text {ext }}\right)$ and maximum excitation power (Pext) of $785 \mathrm{~nm}$ and $19.1 \mathrm{~mW}$, respectively, and bias voltage $\left(\mathrm{V}_{\text {in }}\right)$ of $2.0 \mathrm{~V}$. Because the LD generates thermal energy, thermal energy control is essential. Thus, the LD was connected to a photodetector (PD) that can detect and control the heat generated by the LD. When heat is generated, the control unit of the drive module adjusts to the heat after its detection by the PD.

The Thorlabs MLD203P2 drive module was used, with a bias voltage and current of DC $5 \mathrm{~V}$ and $200 \mathrm{~mA}$, respectively. A variable resistor (max. $10 \mathrm{k} \Omega$ ) was used for laser brightness control; therefore, the LD can adjust the excitation power. An endoscope camera was used in the complementary metal oxide semiconductor (CMOS) sensor. The camera has a bias voltage of $3 \mathrm{~V}$. Its horizontal length and diameter are $4 \mathrm{~mm}$ and 1.5 $\mathrm{mm}$, respectively, as shown in Fig. 3.

The resolution, imaging frame, pixel size, field of view (FOV), and sensor type of the camera are $1600 \times 1200,30 \mathrm{fps}, 2.0 \mathrm{Mpixel}, 60^{\circ}$, and CMOS sensor, respectively. The irradiation beam angle of the LD is free, i.e., $360^{\circ}$. The WD and beam focus angle $\left(\theta_{\mathrm{f}}\right)$ of the camera are $10 \mathrm{~mm}$ and $9.5^{\circ}$, respectively (see Fig. 1). Then, the WD was adjusted from 0 to $50 \mathrm{~cm}$, and the realization environment was measured as $50 \mathrm{~mm}$.

Fig. 3 shows the removal of the infrared (IR)-cut filter from the internal structure of the camera. Fluorescence dye (ICG) imaging could not be achieved because the emission wavelength $(\mathrm{Em})$ of ICG fluorescence dye imaging is in the IR band (above $800 \mathrm{~nm}$ ) [4], [17]-[19]. Thus, the IR band ( $>800 \mathrm{~nm})$ cannot transmit the IR-cut filter in the camera. 


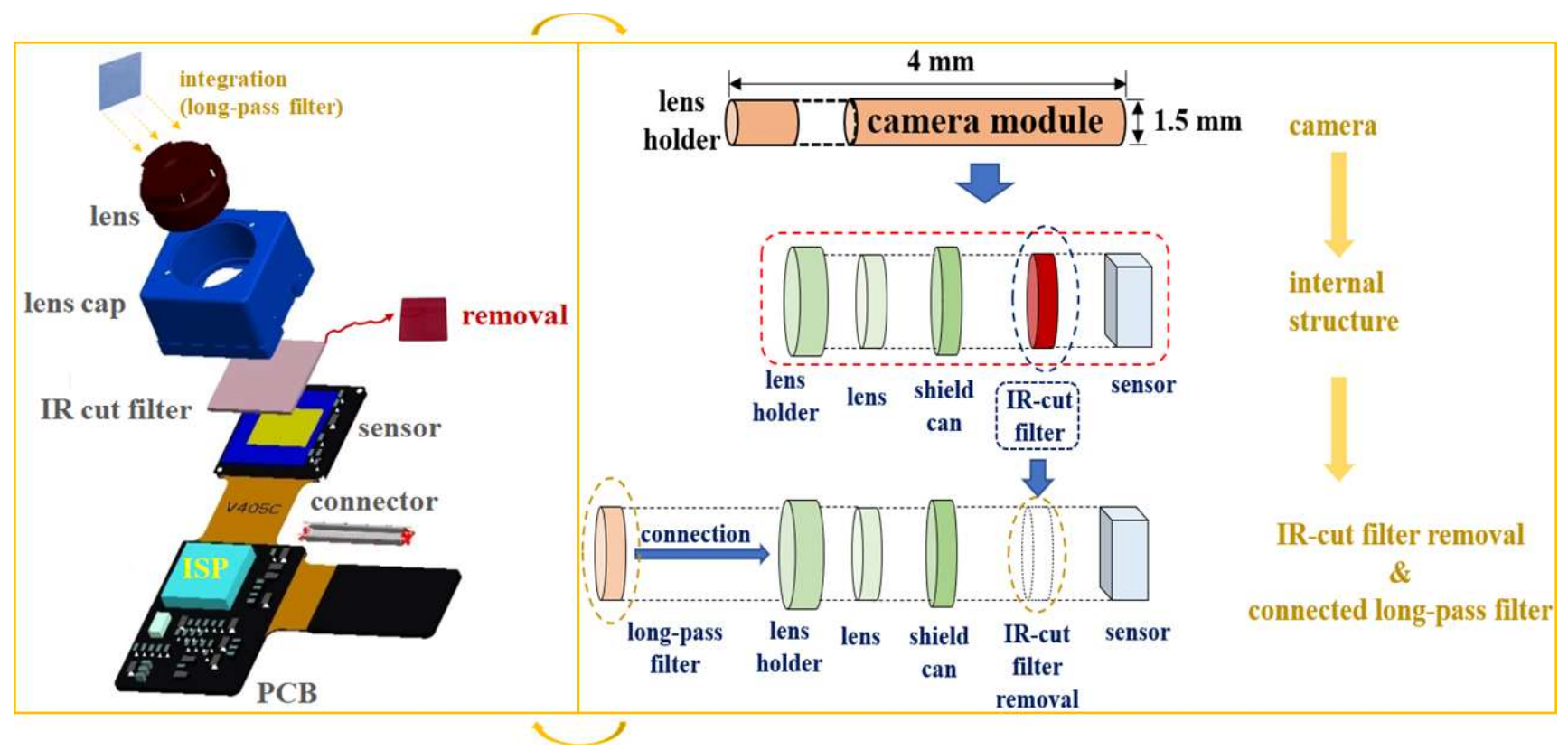

(a)

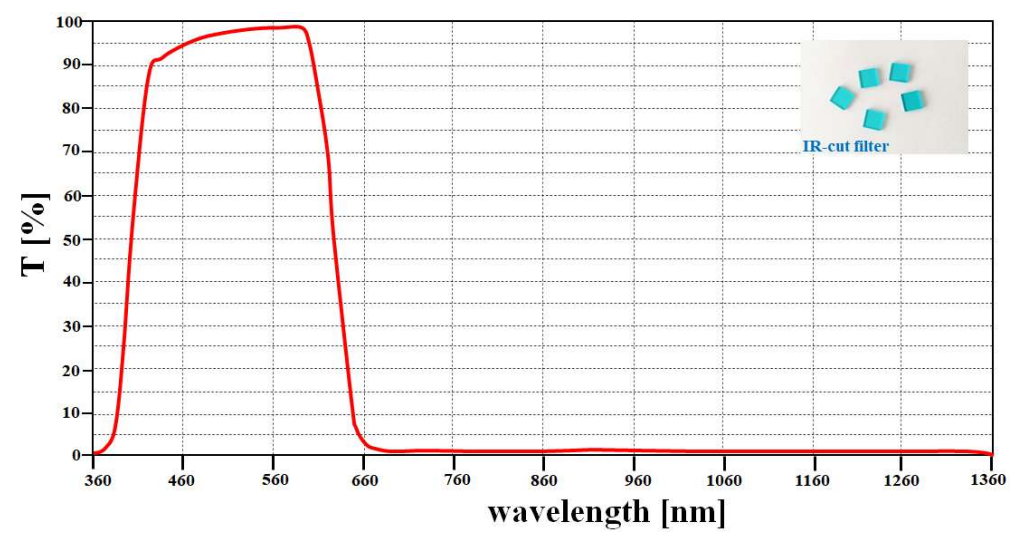

(b)

Figure 3. Fabrication of a camera and experimental results: (a) removed IR-cut filter and connected longpass filter and (b) measurement results of the IR-cut filter.

The camera lens (head) was connected to a longpass filter with a cut-on wavelength of $800 \mathrm{~nm}$. The longpass filter was fabricated using titanium oxide $\left(\mathrm{T}_{\mathrm{i} 3} \mathrm{O}_{5}\right)$, silicon oxide $\left(\mathrm{S}_{\mathrm{i}} \mathrm{O}_{2}\right)$, and a glass-coated substrate, as shown in Fig. 4 . The fabricated filter has a $t, l$, and $\mathrm{h}$ of 2,4 , and $0.4 \mu \mathrm{m}$, respectively; its diameter is $1.5 \mathrm{~mm}$. 


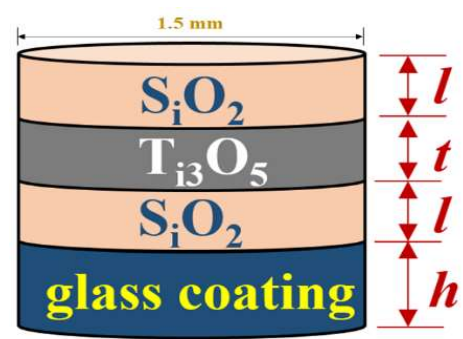

[layout]

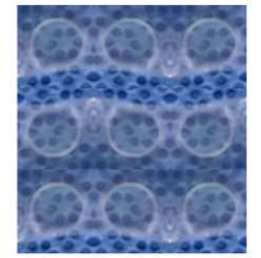

[fabrication]

Figure 4. Structure of the optical longpass filter.

The longpass filter only transmits the wavelengths emitted by the ICG fluorescence dye, and produces a diagnostic image without any excitation wavelength. The pen-type probe was equipped with a low-cost camera. Fig. 5 shows the fabrication of the pen-type probe by $3 \mathrm{D}$ printing.
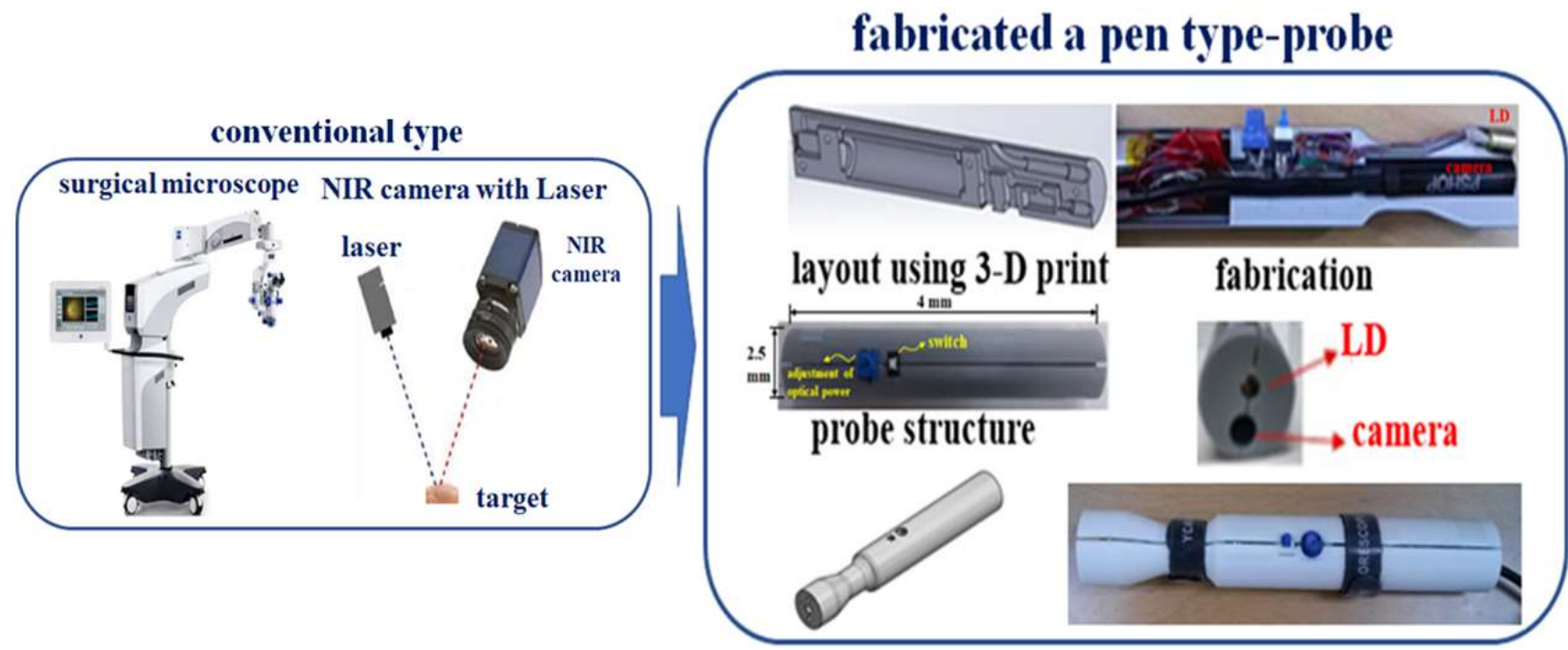

Figure 5. Fabrication of the pen-type probe using a 3D printing technique.

The pen-type probe is lighter than the conventional surgical microscope, with an overall size and weight of $17 \mathrm{~mm} \times 2.5 \mathrm{~mm}$ and $32 \mathrm{~g} \mathrm{(} \pm 2 \mathrm{~g})$, respectively; the conventional surgical microscope weighs $6.0 \mathrm{~kg}$ [20].

\section{Experimental Results}

The measurement results of the fabricated long-pass filter are shown in Fig. 6. The cut-in wavelength, transmission (T), and reflection (OD: optical density) are $805 \mathrm{~nm}, 98 \%$, and $0.2 \%$, respectively. 


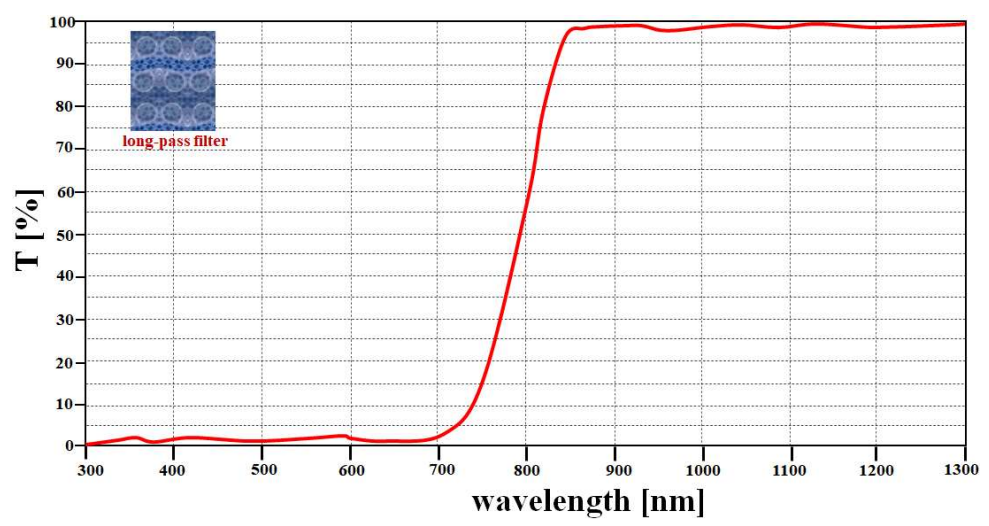

Figure 6. Measurement results of the fabricated long-pass filter.

A small animal (rat) experiment was performed to test the performance of the proposed pen-type probe. Fig. 7 shows the surgical environment for the animal test. As shown in the figure, the pen-type probe is connected to an external monitor by a cable.

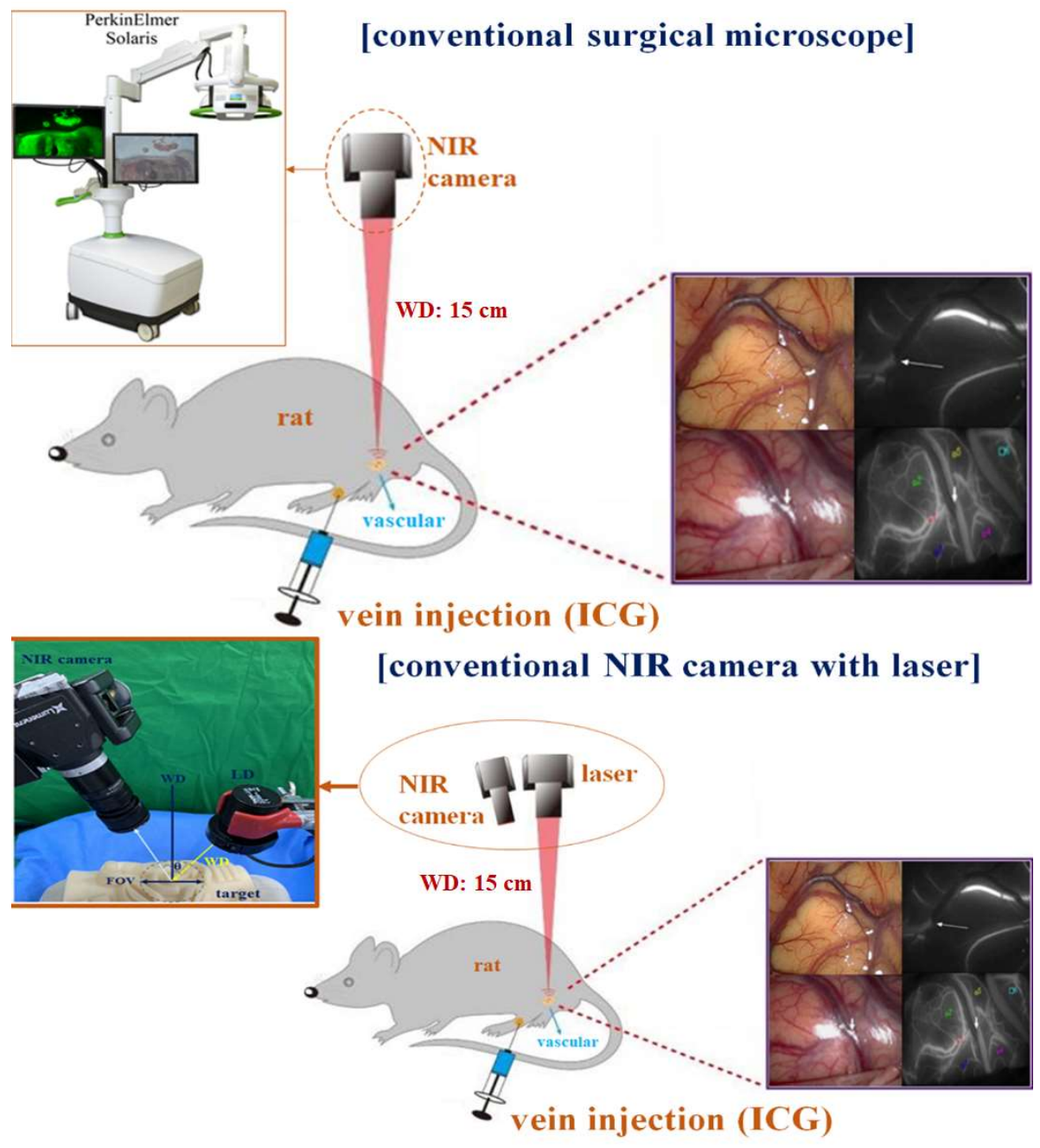

(a) 

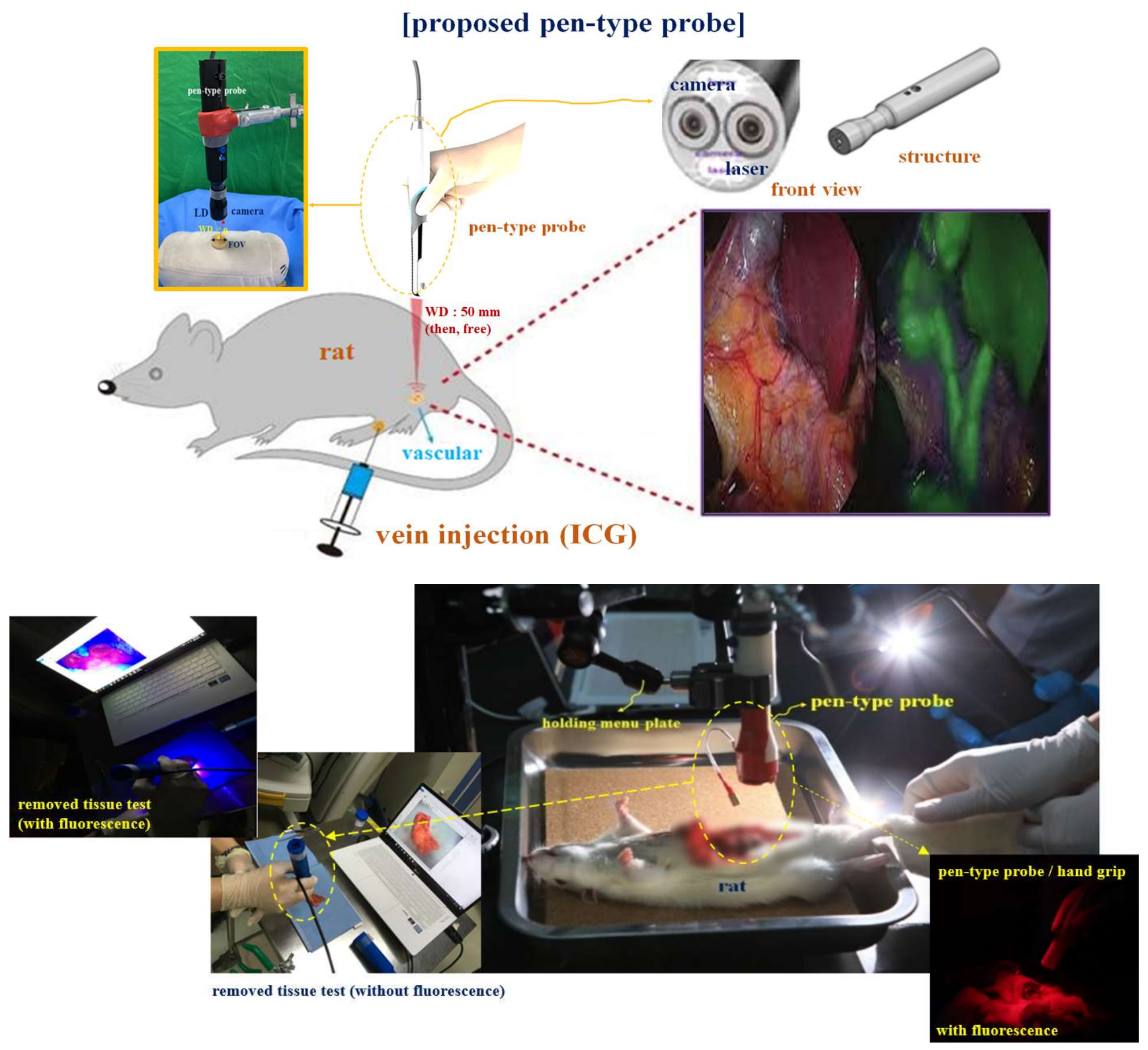

(b)

Figure 7. Rat surgery and small animal test: (a) test using conventional surgical microscope and NIR camera with laser, and (b) pen-type probe test.

The WDs were determined as $15 \mathrm{~cm}$ (conventional surgical microscope and NIR camera) and $50 \mathrm{~mm}$ (pen-type probe). However, the WD of the pen-type probe is free between $50 \mathrm{~mm}$ and $50 \mathrm{~cm}$. The animal was tested at the Experimental Animal Center of the Lee Gil Ya Cancer and Diabetes Research Institute (LCDI-2017-0050) in Korea. We obtained permission from the Animal Institutional Review Board (IRB) of the Animal Ethics Commission. In the animal test, the specimen was a Sprague Dawley rat (male), eight weeks old, weighing $240 \mathrm{~g}$. As shown in Fig. 8(a), an ICG fluorescent contrast agent was injected into the rat vein. The fluorescence expression of the agent was determined according to the concentration. 


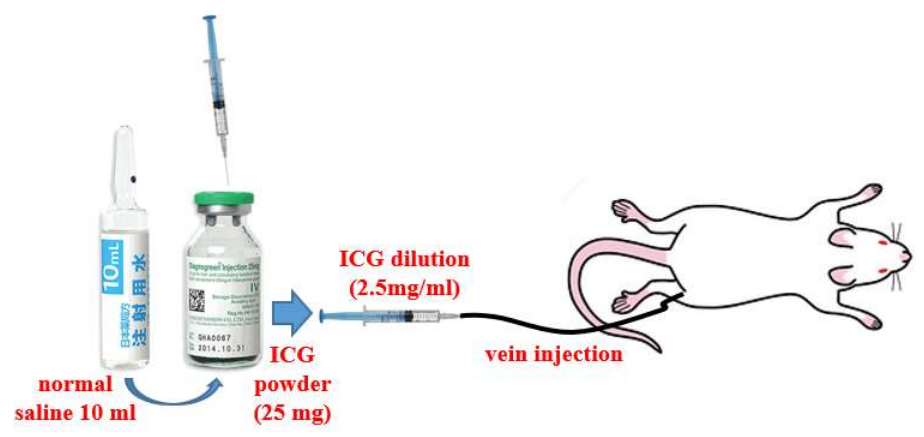

(a)

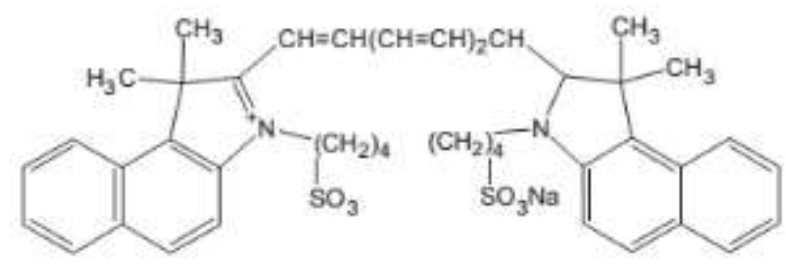

(b)

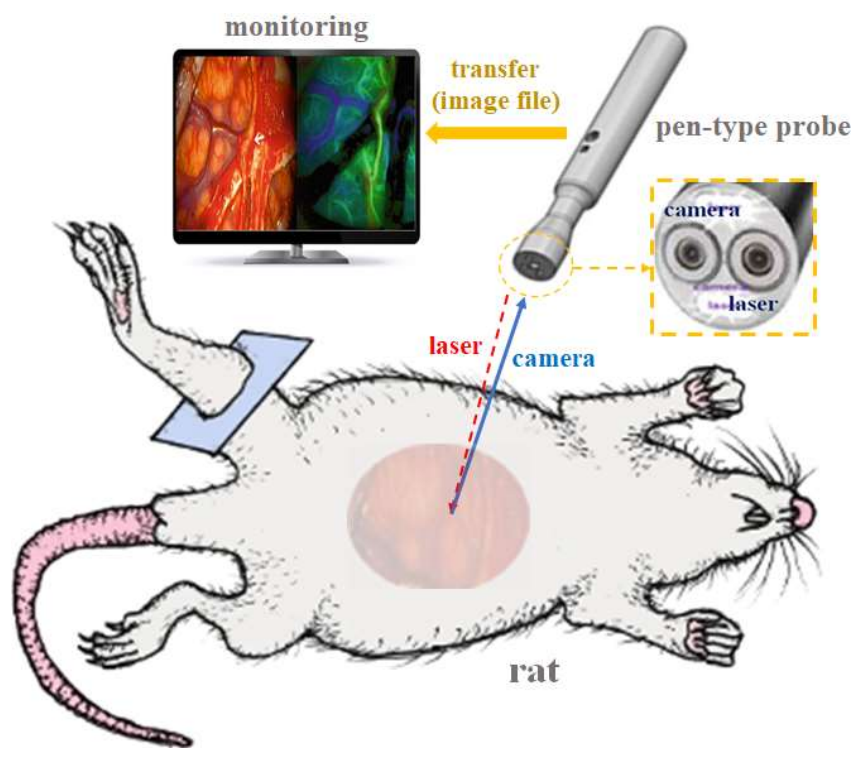

(c)

Figure 8. ICG fluorescence contrast process: (a) process of injection, (b) molecular structure of plasma protein binding by ICG injection, and (c) test method.

The fluorescent contrast agent was mixed with a $10 \mathrm{ml}$ aqueous injection solution and $25 \mathrm{mg}$ of ICG (Dongindang, Republic of Korea, one vial $25 \mathrm{~mL} /$ insurance code: $664100231)$. The mixture $(0.2-0.4 \mathrm{ml})$ was injected into the rat vein by a syringe. The injected ICG fluorescent material binds to plasma protein, as shown in Fig. 8(b) [21]. For the animal test of ICG fluorescence emission, the optical source of the pen-type probe was irradiated to the vasculature of a rat. The excitation wavelength $\left(\lambda_{\text {ext }}\right)$ and laser power with the conventional surgical microscope and NIR camera (Lt $225 \mathrm{NIR} \mathrm{M} / \mathrm{N}$, respectively) were $785 \mathrm{~nm}$ and $60 \mathrm{~mW} / \mathrm{cm}^{2}$, respectively. Then, the laser $(785 \mathrm{~nm})$ was used in the PerkinElmer Solaris optical imaging system and Thorlabs CPS780S (for NIR 
camera). The excitation wavelength $\left(\lambda_{\text {ext }}\right)$ and LD power of the pen-type probe were 785 $\mathrm{nm}$ and $4.0 \mathrm{~mW} / \mathrm{cm}^{2}$, respectively. The LD (Thorlabs L785P90) power was adjusted from $0 \mathrm{~mW} / \mathrm{cm}^{2}$ to $19.0 \mathrm{~mW} / \mathrm{cm}^{2}$. The camera used was the SE-8J200 (endoscopy-type microscopy).

When the LD of the pen-type probe was irradiated with a wavelength of $785 \mathrm{~nm}(4.0$ $\mathrm{mW} / \mathrm{cm}^{2}$ ) to a blood vessel, fluorescence was emitted from the blood vessel and the blood flow state could be observed. The fluorescence emission wavelength and power ranged from 805 to $830 \mathrm{~nm}$ [4], [17]-[19].

Fig. 9 shows the resulting fluorescence emissions in vascular flow experiments on small animals, including the state of blood flow through ICG fluorescence in blood vessels. Fluorescence emission images of blood flow were collected using the NIR and IR cameras. The collected images could be observed through a cable.

The fluorescent contrast agent has a liver uptake phenomenon owing to its half-life of $3.4 \mathrm{~min}$. Then, the [4], [19], [22] of ICG fluorescence has average $2.4 \mathrm{~min}(0.01 \mathrm{mg} / \mathrm{kg})$ liver uptake phenomenon. The observation results (see. Fig. 9) show that the pen-type probe's camera has excellent image resolution compared with the NIR camera.
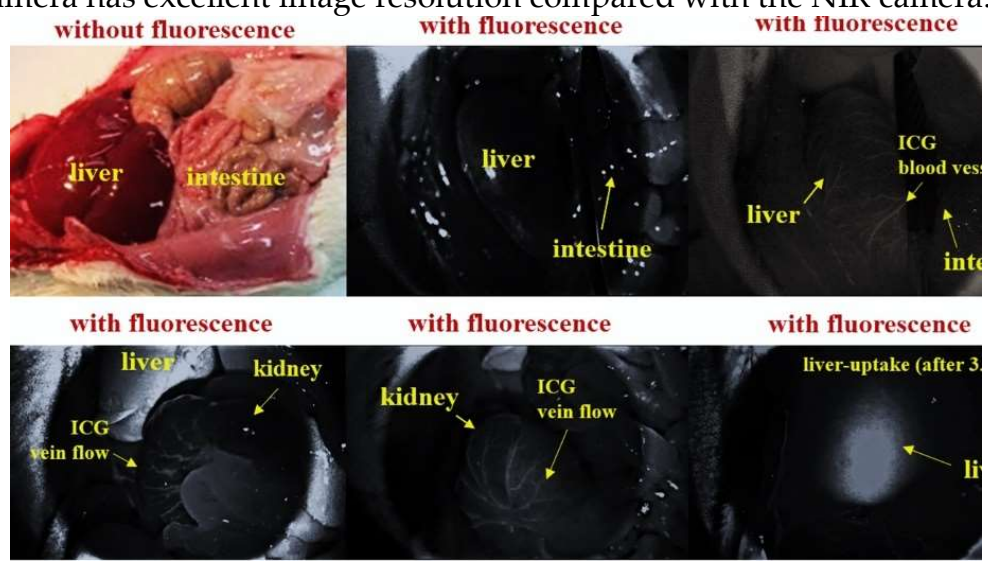

(a)

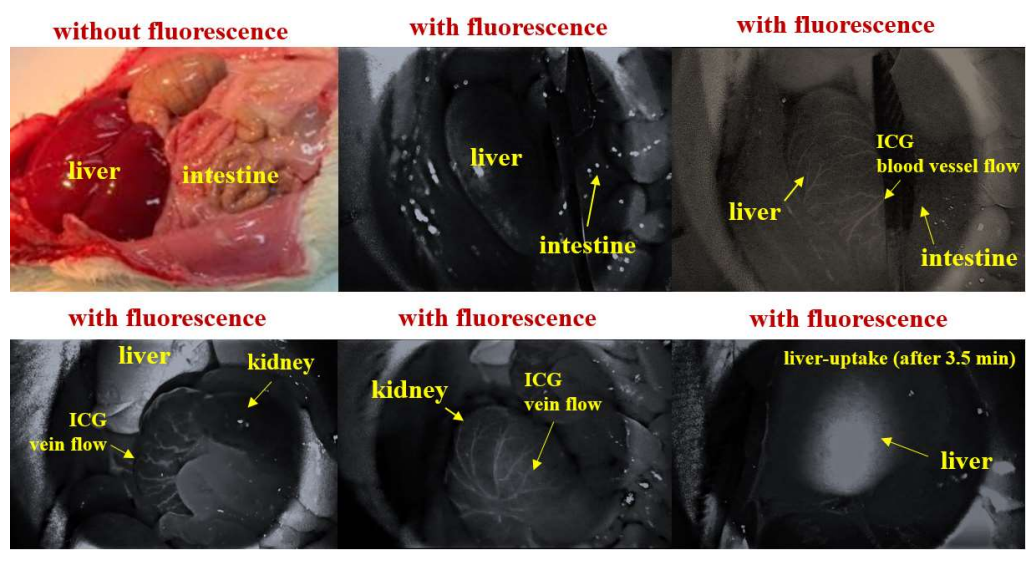

(b) 


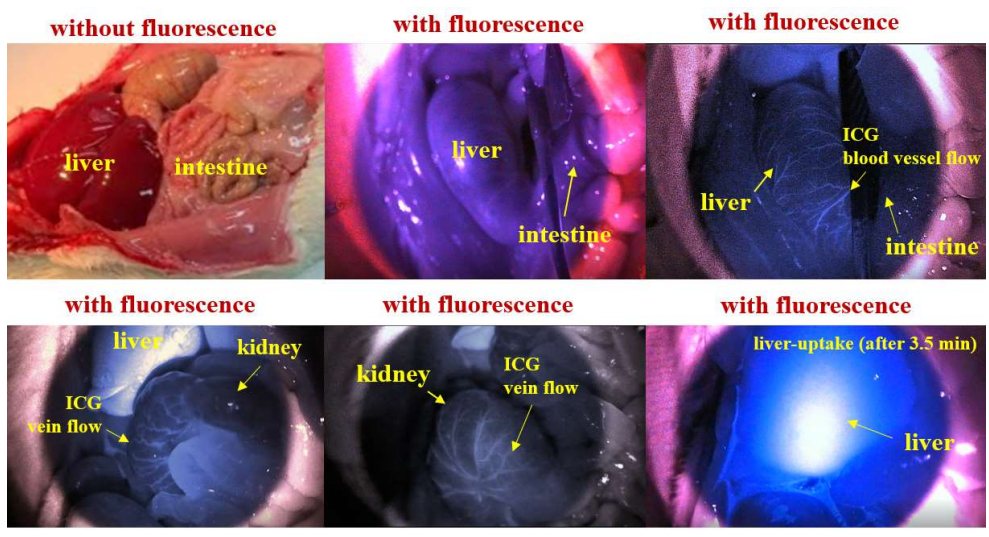

(c)

Figure 9. Experimental results of small animals: (a) conventional surgical microscope, (b) NIR camera (Lt-225 M/N), and (c) pen-type probe camera.

\section{Discussion}

In the observation progress of the ICG fluorescence emission (see Fig. 9), the camera can be compared with the pen-type probe and NIR camera (Lumenera Lt-225 M/N). The NIR camera is suitable black and white and color imaging, while the pen-type probe camera shows color imaging. The pen-type probe's camera appears to be better than the others, as shown in Table 1.

Table 1. Comparison of the performance of the pen-type probe's camera with others.

\begin{tabular}{ccccccc}
\hline & This work & Lt-225 & [18] & $\begin{array}{c}\text { Fluoptics } \\
\text { [18] }\end{array}$ & [20] & [23] \\
\hline $\begin{array}{l}\text { Resolu- } \\
\text { tion [pix- } \\
\text { els] }\end{array}$ & $1600 \times 1200$ & $2048 \times 1088$ & $1024 \times 1024$ & $720 \times 576$ & $800 \times 600$ & $256 \times 512$ \\
$\begin{array}{c}\text { Frame } \\
\text { rate [fps] } \\
\text { FOV } \\
{\left[\mathrm{mm}^{2}\right]}\end{array}$ & 70 & 170 & 30 & 25 & 20 & 20 \\
Sensor & CMOS & CMOS & CMOS & CCD & CMOS & CCD \\
\hline
\end{tabular}

The resolution, frame, and pixel size of the pen-type probe camera (except for the NIR camera) were more than two times better than those in [18], [20], and [23]. In Fig. 10, the external irradiation optical power source (LD) of a conventional surgical microscope is $55-160 \mathrm{~mW} / \mathrm{cm}^{2}$ [4], [15], [18], [23]-[27], while the WD is 15-30 cm [4], [15], [18], [23], [27]. However, the external irradiation optical intensity decreased by approximately $58 \%$ when the WD is above the minimum value of $200 \mathrm{~mm}$ [28].

To generate ICG fluorescence emission, it is necessary to increase the optical source intensity or decrease the WD. If the optical intensity of the LD (Thorlabs, L785PO) is increased, then the LD will cause thermal breakage because of its vulnerability to heat, as proven by the experiment. If the WD of a conventional surgical microscope is decreased, then the FOV dimension narrows. Therefore, the viewing angle for lesion observation also becomes very narrow [29].

The pen-type probe's LD enables the adjustment of the LD power and WD. As shown in Fig. 10, the pen-type probe's LD and WD were adjusted to $4 \mathrm{~mW} / \mathrm{cm}^{2}$ and 50 $\mathrm{mm}$, respectively, while the FOV was obtained as $60^{\circ}$. Thus, the vascular flow condition can be monitored according to ICG fluorescence emission. The conventional surgical 
microscope limits the adjustment range of the optical power source $\left(55-169 \mathrm{~mW} / \mathrm{cm}^{2}\right)$ and WD $(15-30 \mathrm{~cm})$. In contrast, the optical source and WD of the pen-type probe can be freely adjusted. The maximum photographing angle of a conventional surgical microscope is $30^{\circ}$ [30], while that of the pen-type probe is free $\left(360^{\circ}\right)$ [31]-[34]. Therefore, the pen-type probe does not have a blind spot, and the FOV angle is broader than that of the conventional surgical microscope.

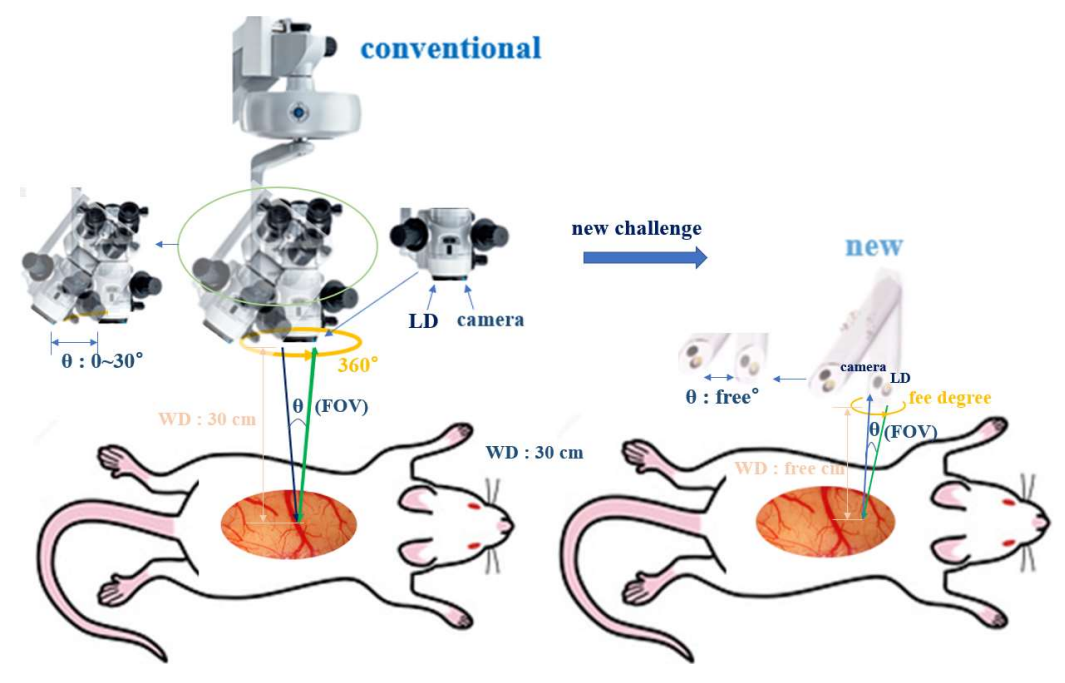

Figure 10. Comparison of the performance of the surgical microscope and pen-type probe.

The conventional surgical microscope is bulky $(6 \mathrm{~kg})$ [20], and requires electrical power supplied by an external source. Thus, it is not portable and occupies a large amount of space in the operating room because of its size. On the other hand, the maximum irradiation power of an LD is $19 \mathrm{~mW}$; the actual LD power used is within 4 $\mathrm{mW}$; and is reduced by approximately 2.89 to 40 times. Thus, the pen-type probe can be powered by a battery. For this reason, it can be reduced to a small size, making it portable and suitable for a narrow operating room.

The characteristics of ICG fluorescence contrast diagnosis have been formally approved by the United States Food and Drug Administration [17, 18]. Therefore, it can be applied to observe the conditions of the heart, liver, blood flow, and blood vessels; and in angiography, cerebrovascular arteriovenous malformation, and vascular suture surgery [18], [23], [28], [35]-[38].

\section{Conclusions}

A pen-type probe is small and portable. It consists of an LD, endoscope camera, brightness control device for the light source, power supply, and communications cable. The pen-type probe can be connected to an external monitor via cable. Therefore, it can be used to observe tissues from the outside through a monitor.

With the use of a pen-type probe, the shape, color, and boundary of tumors and vasculature can be easily distinguished through fluorescence; the beam direction and angle can also be adjusted. Therefore, free organizational observations are possible.

Pen-type probes are independent of the WD. Thus, there is neither an optical density loss nor a blind spot. Laser irradiation should be conducted within a safe range of tissues, and in accordance with international medical standards (IEC 60601-2-41). Small animal experiments were conducted at the Experimental Animal Center of the Lee Gil Ya Cancer Diabetes Research Institute in Korea (IACUC No. LCDI-2017-0050).

In these experiments, the wavelength and power of the LD were $785 \mathrm{~nm}$ and 4.0 $\mathrm{mW} / \mathrm{cm}^{2}$, while the fluorescence emission wavelength and power were $805 \mathrm{~nm}$ and 0.2 $\mathrm{mW} / \mathrm{cm}^{2}$, respectively. The maximum fluorescence brightness duration was $3.4 \mathrm{~min}$. 
Author Contributions: design and simulation; K. C. Yoon, analysis, and supervisor; KG. Kim, and clinical data collection, S. H. Lee. All authors have read and approved the published version of the manuscript.

Funding: This work was supported by the Technology Innovation Program (K_G012001187801, "Development of Diagnostic Medical Devices with Artificial intelligence Based Image Analysis Technology") funded By the Ministry of Trade, Industry \& Energy (MOTIE, Korea)“.

Institutional Review Board Statement: Not applicable.

Informed Consent Statement: Not applicable.

Data Availability Statement: The data presented in this study are available upon request from the corresponding author. The data are not publicly available owing to privacy and ethical restrictions.

Acknowledgments: The animal test was conducted at the Experimental Animal Center of Korea's Lee Gil Ya Cancer Diabetes Research Institute (IACAU No.: LCDI-2017-0050) with permission from the Animal Institutional Review Board (IRB) of the Animal Ethics Commission.

Conflicts of Interest: The authors declare no conflict of interest.

\section{References}

1. F. Lefranc, N. Sadeghi, I. Camby, T. Metens, O. Dewitte, and R. Kiss. Present and potential future issues in glioblastoma treatment. Expert Rev. Anticancer Therapy, 2006; 6, 719-32. [doi: 10.1586/14737140.6.5.719.]

2. W. Stummer, A Novotny, H. Stepp, C. Goetz, K. Bise, and H. J. Reulen. Fluorescence-guided resection of glioblastoma muliforme utilizing 5-ALA-induced porphyrins: a prospective study in consecutive patients. J. Neurosurgery, 2000; 93, 1003-13. [doi: 10.3171/jns.2000.93.6.1003. [doi: 10.3171/2014.3.JNS12578]

3. D. I. Pitskhelauri, A. N. Konovalov, G. A. Shekutev, N. B. Rojnin, I. A. Kachkov, D. Y. Samborskiy, A. Z. Sanikidze, and D. N. Kopachev. A novel device for hands-free positioning and adjustment of the surgical microscope. J. Neurosurg. 2014; 161-164. [doi: 10.3171/2014.3.JNS12578]

4. A. Dupréea, H. Rießa, C. Detter, E. S. Debus, and S. H. Wipper. Utilization of indocynanine green fluorescent imaging (ICG-FI) for the assessment of microperfusion in vascular medicine. Innov. Surg. Sci; 2018, 3, 193-201. [doi: 10.1515/iss-2018-0014]

5. A. V. D. Souza, H. Lin, E. R. Henderson, K. S. Samkoe, and B. W. Poguea,d. Review of fluorescence guided surgery systems: identification of key performance capabilities beyond indocyanine green imaging. J. of Biomedical Optics, 2016 ; $21,080901$. [doi: 10.1515/iss-2018-0014.]

6. C. Malkanthi and M. B. Dissanayake. Brain tumor boundary segmentation of MR imaging using spatial domain image processing. Int. J. for Innovation Education and Research, 2017; 5, 01-09. [doi: https://doi.org/10.31686/ijier.vol5.iss10.621]

7. N. Unno, M. Suzuki, N. Yamamoto, K. Inuzuka, D. Sagara, M. Nishiyama, H. Tanaka, and H. Konno. Indocyanine green fluorescence angiography for intraoperative assessment of blood flow: a feasibility study. European J. Vasc. Endovasc. Surg., 2008; 35, 205-207. [doi.org/10.1016/j.ejvs.2007.09.001]

8. T. Desmettre, J. M. Devoisselle, and S. Mordon. Fluorescence properties and metabolic features of indocyanine green (ICG) as related to angiography. Elsevier Sci. Inc., 2000; 45, pp. 15-27. [doi: 10.1016/s0039-6257(00)00123-5]

9. Handgraaf H, Boonstra M, Erkel A Van. Current and future intraoperative imaging strategies to increase radical resection rates in pancreatic cancer surgery. Biomed Res., 2014; pp. 2014890230. [doi: 10.1155/2014/890230]

10. B. Qi, A. J. Crawford, N. E. Wojtynek, M. B. Holmes, J. J. Souchek, G. Almeida-Porada, Q. P. Ly, S. M. Cohen, M. A. Hollingsworth, and A. M. Mohs. Indocyanine green loaded hyaluronan-derived nanoparticles for fluorescence-enhanced surgical imaging of pancreatic cancer. Nanomedicine: Nanotechnology, Biology, and Medicine, 2018; 14, 769-780. [10.1016/j.nano.2017.12.015]

11. T. Wada, K. Kawada, R. Takahashi, M. Yoshitomi, K. Hida, S. Hasegawa, Y. Sakai. ICG fluorescence imaging for quantitative evaluation of colonic perfusion in laparoscopic colorectal surgery. Surg. Endosc., 2017; 31, 4184-4193. [doi: 10.1007/s00464-017-5475-3]

12. G. M. Son, M. S. Kwon, Y. Kim, J. Kim, S. H. Kim, and J. W. Lee. Quantitative analysis of colon perfusion pattern using indocyanine green (ICG) angiography in laparoscopic colorectal surgery. Surgical Endoscopy, 2019; 33, 1640-1649 [doi:10.1007/s00464-018-6439-y].

13. J. C. Delong, E. P. Ward, T. M. Lwin, K. T. Brumund, K. J. Kelly, S. Horgan, and M. Bouvet. Indocyanine green fluorescence-guided parathyroidectomy for primary hyperparathyroidism. Surgery; 2018, 163, 388-392. [doi.org/10.1016/j.surg.2017.08.018]

14. J. C. Yoo, S. P. Lee, J. G. Kim, G. H. Choi, H. T. Yeo. An image study of malignant glioma model with diffraction enhanced imaging computed tomography. J. Korean Brain Tumor Society, 2011; 10, 103-109.

15. N. Y. Hong, H. R. Kim, H. M. Lee, D. K. Sohn, and KG Kim. Fluorescent property of indocyanine green (ICG) rubber ring using LED and laser light sources. Biomedical Optics Express, 2016; 7, 1637-1644. [doi.org/10.1364/BOE.7.001637] 
16. C. T. Wen, Y. Y. Liu, H. Y. Fang, M. J. Hsieh, and Y. K. Chao. Image-guided video-assisted thoracoscopic small lung tumor resection using near-infrared marking. Springer Surg. Endosc., 2018; 32, 4673-4680.

17. J. T. Alander, I. Kaartinen, A. Laakso, T. Patila, T. Spillmann, V. V. Tuchin, M. Venermo, and Petri Välisuo. A review of indocyanine green fluorescent imaging in surgery. Hindawi Int. J. Biomedical Imaging, 2012; 2012, 1-26. [doi.org/10.1155/2012/940585]

18. A. V. Dsouza, H. Lin, E. R. Henderson, K. S. Samkoe, and B. W. Pogue. Review of fluorescence guided surgery systems: identification of key performance capabilities beyond indocyanine green imaging. J. of Biomedical Optics, 2016; 21, 08090(1) 080901. (15). [doi.org/10.1117/1.JBO.21.8.080901]

19. GR Cherrick, SW Stein, CM Leevy, CS Davidson. Indocyanine green: Observation on its physical properties, plasma decay and hepatic extraction. J. of Clin Invest., 1960; 39, 592-600. [doi: 10.1172/JCI104072]

20. J. R. Watson, C. F. Gainer, N. Martirosyan, J. Skoch, G. Michael Lemole, R. Anton, and M. Romanowski. Augmented microscopy: real-time overlay of bright-field and near-infrared fluorescence images. J. of Biomedical Optica, 2015; $20,106002$. [doi: 10.1117/1.JBO.20.10.106002]

21. M. Miwa. The principle of ICG fluorescence method. The Open surgical Oncology Journal, 2010; 2, 26-28. [doi: 10.2174/1876504101002010026]

22. M. Hope-Ross, LA Yannuzzi, E. S. Gragoudas, D. R. Guyer, J. S. Slakter, and J. A. Sorenson. Adverse reactions due to indocyanine green. Ophthalmology, 1994; 101, 529 (33). [doi: 10.1016/s0161-6420(94)31303-0]

23. L. Ma and B. Li. Comprehensive review of surgical microscopes: technology development and medical applications. J. of Biomedical Optics, 2021; 26, pp. 010901 (1). [doi: 10.1117/1.JBO.26.1.010901]

24. C. Detter, S. Wipper, D. Russ, A. Iffland, L. Burdorl, and E. Thein. Fluorescent cardiac imaging: a novel intraoperative method for quantitative assessment of myocardial perfusion during graded coronary artery stenosis. Circulation, 2007; 116, 1007-14. [doi: 10.1161/CIRCULATIONAHA.106.655936]

25. S. Wipper, B. Reiter, D. Russ, F. Hahnel, JF Kersten, and T. Kolbel. Distinction of non-ischemia inducing versus ischemia inducing coronary stenosis by fluorescent cardiac imaging. Int. J. Cardiovasc Imaging, 2016; 32, 363-71. [doi: 10.1007/s10554-015-0767-y]

26. C. Detter, D. Russ, JF Kersten, H. Reichenspurner, and S. Wipper. Qualitative angiographic and quantitative myocardial perfusion assessment using fluorescent cardiac imaging during graded coronary artery bypass stenosis. Int. J. Cardiovasc Imaging 2018; 34, 159-167. [doi: 10.1007/s10554-017-1212-1]

27. K. C. Yoon, EJ. Kim, KG. Kim, and SH Lee. A multi-detection microscope with 5-ALA and ICG Using dual array LED. Current Optics Photonics, 2019; 3, 256-262.

28. P. Hoerenz, The operating microscope I. Optical principles illumination systems, and support systems. J. of Microsurgery, 1980; 1, 364-369. [doi: 10.1002/micr.1920010506]

29. P. Hoerenz. The operating microscope II. Individual parts, handling, assembling, focusing, and balancing. J. of Microsurgery, 1980; 1,419-427. [doi: 10.1002/micr.1920010603]

30. J. Lee, R. E. Wijesinghe, D. Jeon, P. Kim, Y. H. Choung, J. H. Jang, M. Jeon, and J. Kim. Clinical utility of intraoperative tympanomastoidectomy assessment using a surgical microscope integrated with an optical coherence tomography. Scientific Reports, 2018; 8, 1-8.

31. F. Ricci and F. Missiroli, L. Cerulli. Indocyanine green dye enhanced micro pulsed diode laser: a novel approach to subthreshold RPE treatment in a case of central serous chorioretinopathy. European J. Ophthalmology, $2003 ; 14,74-82$. [doi: 10.1177/112067210401400115]

32. M. V. Marshall, J. C. Rasmussen, I.-C. Tan, M. B. Aldrich, K. E. Adams, X. Wang, C. E. Fife, E. A. Maus, L. A. Smith, E. M. Sevick-Muraca. Near-infrared fluorescence imaging in humans with indocyanine green: a review and update. Open Surgical Oncology Journal (Online), 2010; 2, 12. [doi: 10.2174/1876504101002010012]

33. G. Themelis, J. S. Yoo, V. Ntziachristos. Multispectral imaging using multiple-bandpass filters. Optics Lett., 2008; 33, 1023-1025. [doi: 10.1364/ol.33.001023]

34. Y. Kondo, Y. Murayama, H. Konishi, R. Morimura, S. Komatsu, A. Shiozaki, Y. Kuriu, H. Ikoma, T. Kubota, M. Nakanishi. Fluorescent detection of peritoneal metastasis in human colorectal cancer using 5-aminolevulinic acid. Int. J. Oncology, 2014; 45, pp. 41-46. [doi: 10.3892/ijo.2014.2417]

35. G. Klein, R. Baumgartner, R. Flower. An image processing approach to characterizing choroidal blood flow. Investigative Ophthalmology \& Visual Science, 1990; 31, 629-637.

36. G. Themelis, J. S. Yoo, K.-S. Soh, R. Schulz, V. Ntziachristos. Real-time intraoperative fluorescence imaging system using light-absorption correction. J. Biomedical Optics, 2009; 14, 064012-064012-064019. [doi.org/10.1117/1.3259362]

37. A. Rück, C. Hauser, S. Lorenz, S. Mosch, S. Rotte, M. Kessler, S. Kalinina. Cell metabolism, tumour diagnosis and multispectral FLIM. In SPIE BiOS. Int. Society for Optics and Photonics, San Francisco, California, USA, 2013; pp. 85880U-85880U-85888. [doi.org/10.1117/12.2003729] 